\title{
A new class of vacillations of the stratospheric polar vortex
}

\author{
R. K. Scott \\ School of Mathematics and Statistics, University of St Andrews, St Andrews, KY16 9SS, UK \\ ${ }^{*}$ Correspondence to: R. K. Scott, School of Mathematics and Statistics, University of St Andrews, North Haugh, \\ St Andrews, KY16 9SS, UK
}

\begin{abstract}
A new class of persistent vacillations of the winter polar vortex, under the action of topographic wave forcing and radiative cooling, is identified in numerical integrations of the rotating shallow water equations. The vacillations are obtained provided only that care is taken to prevent the unconstrained growth of tropical easterlies that otherwise develop as the result of persistent angular momentum deposition at low latitudes. The vacillation cycle involves purely barotropic dynamics and is characterized by a dynamically controlled rapid splitting and rapid reformation of the vortex followed by a more gradual period of vortex intensification under the influence of radiative relaxation. The onset of the splitting occurs when the frequency of the free mode of the vortex approaches that of the forcing and resembles a resonant excitation. Experiments with an alternative basic state suggest that the vacillations are a robust feature of the topographically forced and radiatively relaxed vortex. In contrast to the behavior found in models with vertical structure, the period of the vacillation cycles here increases with increasing forcing amplitude. A wide range of forcing amplitude exists over which the vortex exhibits distinct regime transitions between a strong, vacillating state and a state in which the vortex is weak and the zonal mean polar flow nearly zero. Comparison with observational reanalysis suggest that the vacillation cycles obtained here may be relevant to the dynamics of some sudden warming events and that the onset of a radiatively dominated regime may be usefully linked to the loss of vortex area following such an event. Copyright (c) 2013 Royal Meteorological Society
\end{abstract}

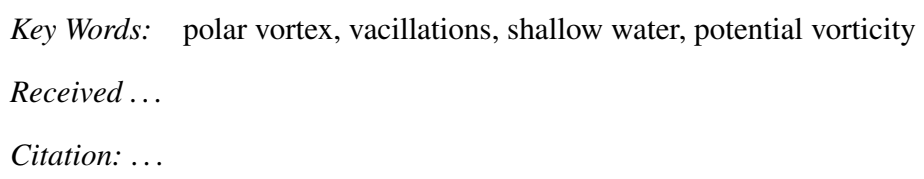

\section{Introduction}

Persistent vacillations of the winter polar vortex involving upward wave propagation, vortex deceleration, and gradual vortex reformation under the action of radiative relaxation have been an area of study since the pioneering work of Holton and Mass (1976). Early work considered vacillations in quasigeostrophic models that were highly truncated in the zonal and meridional directions (Holton and Dunkerton 1978; Chao 1985; Yoden 1987, 1990). These were gradually extended to include more realistic horizontal structure in global primitive equation models (Christiansen 1999; Scott and Haynes 2000; Scott and Polvani 2006). In all of these studies the importance of vertical structure has been emphasized: following the destruction of the vortex, weak low-level winds act to prevent subsequent vertical Rossby wave propagation, allowing the vortex to reform under the influence of radiative relaxation, initially at upper levels and then progressively lower. When low-level westerlies are restored to the point where upward wave propagation resumes, the dynamical forcing of the vortex initiates a new cycle.

In a seperate study, Rong and Waugh (2004) considered similar vacillation experiments in a global shallow water model. While vacillations occurred in their model, they were found not to persist over the same arbitrary long time intervals as their primitive equation counterparts. In particular, the persistent growth of negative (easterly) winds 
across a widening tropical belt eventually encroached far enough into mid-latitudes to impinge on the dynamics of the polar vortex, essentially extinguishing the vortex vacillations after a few cycles. Their study was extended by MirRokni et al. (2011), who found that the shallow water vacillation cycles obtained by Rong and Waugh became weaker and less pronounced at higher resolution and over longer time intervals. They concluded that no persistent vacillations exist in shallow water, again due in part to the build up of tropical easterlies, and that the vacillations obtained by Rong and Waugh were essentially a remnant of initial transience of the integrations resulting from the nonzero initial conditions and relatively rapid initial growth of the topographic forcing. More recently, analysis of the barotropic sudden warming in a global shallow water model (Liu and Scott 2015) hinted at the existence of vacillation regimes in a quasi-stationary context, although integrations of that model over longer times again suffered the effects of unconstrained growth of equatorial easterlies present in Rong and Waugh.

In light of the above studies, it is interesting to ask whether persistent, or stationary vacillations may arise in the shallow water model if the tropical easterlies are prevented from growing to unrealistic magnitudes. Although the persistent vacillation cycles found in primitive equation models appear to rely on a non-trivial vertical structure, with weak low-level winds acting to reduce the upward flux of waves during the vortex recovery period, the vortex break-down phase of the cycles, in contrast, appears to be largely barotropic (see, e.g. Fig.6 of Scott and Polvani (2006)). This suggests that propagating waves may not be playing an important role throughout the entire cycle and that some aspects of internal vacillations may be captured in the shallow water model, as originally suggested by Rong and Waugh. In this regard, we note that there are two separate ways in which vertical structure may be important in the vacillation: the first through the action of vertical propagating waves, the second through the vertical structure of the basic state on which the waves are defined.

The growth of tropical easterlies in the studies of Rong and Waugh (2004) and MirRokni et al. (2011) occurs because radiative relaxation only weakly constrains angular momentum at low latitudes. Mountain torque, the back reaction of the topography on the mean flow, provides a persistent source of negative angular momentum throughout the simulation, which is difficult to balance by the effects of radiative relaxation alone. Although radiative relaxation is not strictly angular momentum conserving in the shallow water system, its constraint on the midlatitude zonal mean flow through thermal wind balance weakens towards the equator due to the reduction in the Coriolis term. As a result, there is a tendency for the negative angular momentum input by the mountain torque to increase predominantly in low latitudes, manifesting as a persistent growth of tropical easterlies. The easterlies are eventually limited as the tropical zone encroaches further into mid latitudes and as the mid-latitude flow adjusts to a state in which the mountain torque no longer provides an effective source of negative angular momentum.

In this paper, we return to the analysis of the topographically forced and radiatively relaxed polar vortex in the shallow water equations, but make explicit efforts to constrain the unrealistic growth of tropical easterlies. A simple expedient is to apply a weak relaxation on the zonally averaged momentum in the tropics and in the hemisphere opposite to the one of interest, as described in section 2 below. Momentum relaxation is absent from the winter hemisphere extratropics of interest. This relaxation on the tropical zonal flow imposes a constraint on the total angular momentum that permits a statistical stationary state to be reached quickly, one, moreover, in which the tropical flow has more realistic magnitudes than otherwise. The equations may then be integrated for arbitrary long times (computing resources permitting). It turns out that, under these conditions, vacillating regimes arise quite robustly in the shallow water model, with dynamical characteristics differing from those previously observed in full primitive equation models. In particular, vacillation cycles typically consist of a rapid destruction of the vortex, followed by a rapid partial recovery involving vortex merger, in turn followed by a gradual strengthening of the vortex under radiative relaxation. The details of the evolution are discussed further in section 3 below.

\section{Model and experimental design}

\subsection{Physical model}

We solve the rotating shallow water equations in vorticitydivergence form:

$$
\begin{array}{r}
\zeta_{t}=-\nabla \cdot\left(\mathbf{u} \zeta_{a}\right) \\
\delta_{t}=-\frac{1}{2} \nabla^{2}|\mathbf{u}|^{2}+\mathbf{k} \cdot \nabla \times\left(\mathbf{u} \zeta_{a}\right)-g \nabla^{2}\left(h+h_{b}\right) \\
h_{t}+\nabla \cdot(h \mathbf{u})=-\alpha\left(h-h_{e}\right) .
\end{array}
$$

Here $\zeta, \delta$ and $h$ are the relative vorticity, divergence, and layer thickness, respectively; $\zeta_{a}=2 \Omega \sin \phi+\zeta$ is the absolute vorticity, where $\Omega$ is the planetary rotation rate and $\phi$ is latitude; $g$ is the gravitational acceleration; $\mathbf{u}=(u, v)$ is the horizontal velocity. The system is forced by a specified topography, $h_{b}$, and a relaxation to an equilibrium height profile $h_{e}(\phi)$ at rate $\alpha$ (see section 2.2 below).

Following Liu and Scott (2015), we use a mean layer depth of $H=10000 \mathrm{~m}$, which gives a shallow water polar Rossby deformation length $L_{D}=\sqrt{g H} / 2 \Omega$ of $\approx$ $2100 \mathrm{~km}$, approximately equal to the Rossby deformation length of the Lamb mode in the winter stratosphere, $N D / f \sqrt{\kappa(1-\kappa)}$, where $D$ is a density scale height and $\kappa=2 / 7$.

The equations are solved in a spherical coordinate system using a standard pseudo-spectral model in which the model fields are represented by a basis of spherical harmonic functions and the nonlinear terms are computed on a physical space grid. The numerical implementation is described in Rivier et al. (2002) and Scott et al. (2004). Time-stepping is via a semi-implicit leap-frog scheme. For numerical stability, a weak, scale-selective dissipation is included on the right-hand side of $1 \mathrm{a}-1 \mathrm{c}$ to prevent the build up of enstrophy at small scales; here we use a dissipation of the form $\nabla^{8}$ with a damping rate of approximately 10/day at the smallest resolved wavenumber. In the experiments reported below we use a triangular spectral truncation with maximum total wavenumber 170 (T170); some individual model integrations were repeated at a resolution of T341. No significant differences were found between integrations at T170 and T341 and we conclude that our results are insensitive to numerical resolution. 

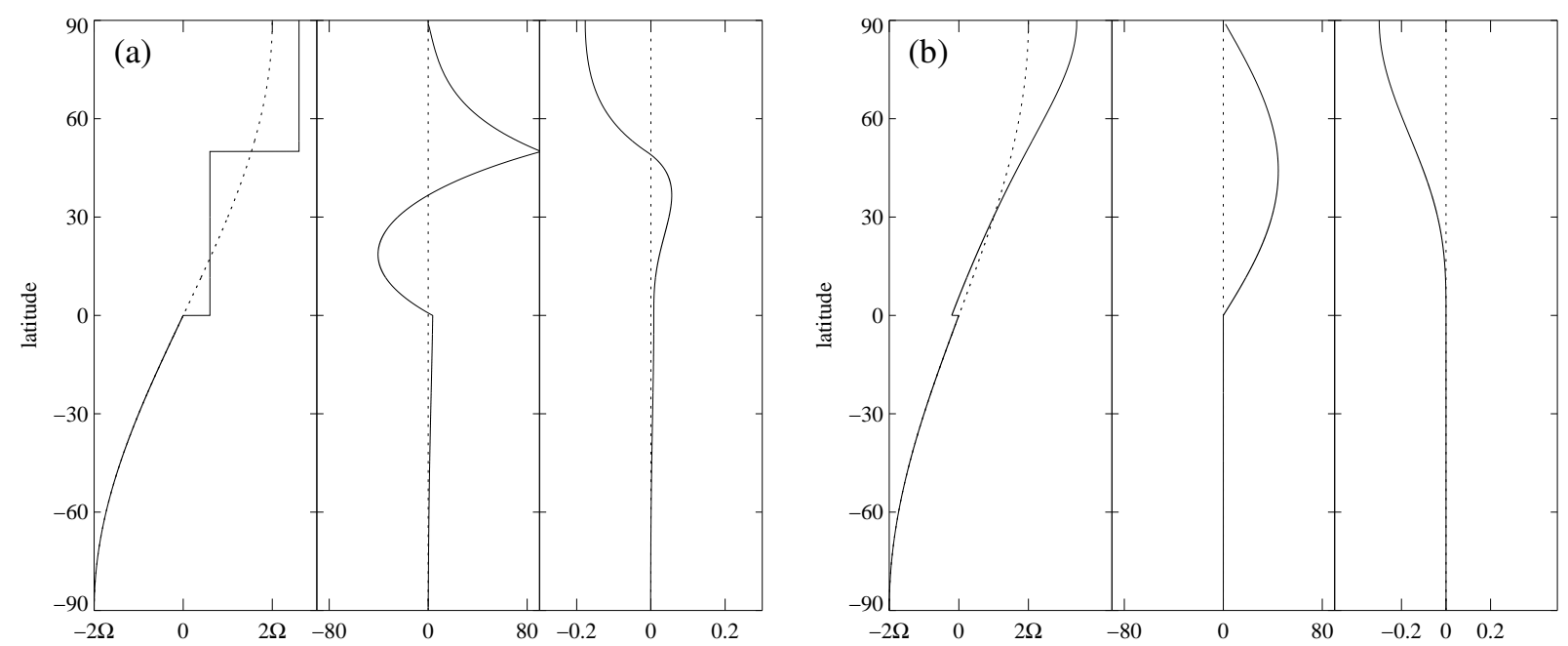

Figure 1. Equilibrium profiles for the vortex patch (a) and broad vortex (b). Left to right in each panel: potential vorticity, $q$, zonal velocity, $u$, in ms ${ }^{-1}$, and height perturbation $h_{e} / H-1$.

\subsection{Radiative equilibrium}

The term on the RHS of (1c) provides a simple representation of the effects of long-wave cooling and a restoring force towards the specified equilibrium height profile, $h_{e}$. The associated mass source or sink may be regarded as a secondary circulation driven by the damping effect on the waves and the associated potential vorticity fluxes (Thuburn and Langneau 1999). In the experiments discussed below, we use a relaxation rate of $1 / 20$ days, representative of the middle stratosphere.

To demonstrate the robustness of the shallow water vacillations we perform experiments with two very different equilibrium profiles $h_{e}(\phi)$. The first of these is the height field in gradient wind balance with a vortex patch of uniform potential vorticity, $q_{v}$, situated over the pole, with vortex edge at $\phi_{1}$ and surrounded by a surf zone of uniform potential vorticity $q_{s}$ :

$$
q_{0}= \begin{cases}q_{v} & \phi_{1} \leq \phi \leq 90^{\circ} \\ q_{s} & 0 \leq \phi<\phi_{1} \\ 2 \Omega \sin \phi & -90^{\circ} \leq \phi<0^{\circ}\end{cases}
$$

In the hemisphere opposite the vortex the potential vorticity takes the planetary value. In the following we take $\phi_{1}=$ $50^{\circ}$, a lower latitude than Liu and Scott (2015) to give a more realistic climatological vortex edge location under perpetual wave forcing. As in Liu and Scott, we choose the value of the potential vorticity jump at the vortex edge $q_{v}-q_{s}=2 \Omega / H$ as a convenient value that results in a maximum zonal velocity of approximately $90 \mathrm{~ms}^{-1}$. The value $q_{s}=0.2933 \times 2 \Omega / H$ is determined as the unique value that satisfies the requirement that the integral of the relative vorticity $\zeta$ over the sphere vanishes. To avoid unwanted Gibbs effects due to the spectral representation of the discontinuity at $\phi=0$ and $\phi=\phi_{1}$, a weak smoothing is applied to (2), as described in the appendix of Liu and Scott. The smoothing has virtually no effect on the overall character of the model evolution.

For $q_{0}$ thus defined, $h_{e}$ is obtained from $1 \mathrm{~b}$ assuming axisymmetry, vanishing $\delta$, and no topography, by the iterative method described in Scott and Liu (2015). The potential vorticity and the associated zonal wind and height profiles are as shown in Fig. 1a.
The second equilibrium height profile is defined as

$$
h_{e}= \begin{cases}H-h_{0} \sin ^{3} \phi & \phi>0 \\ H & \phi<0\end{cases}
$$

which gives a profile very similar to that used in Rong and Waugh (2004). We choose $h_{0}=0.3 H$ as a suitable amplitude that results in a climatological vortex with winds around $90 \mathrm{~ms}^{-1}$ at the vortex edge. The equilibrium height profile and the corresponding balanced zonal velocity and potential vorticity are shown in Fig. 1b. Overall, the character of the second equilibrium state, in particular the zonal velocity and potential vorticity, has a much broader structure than that of the vortex patch. Below, we discuss mainly the results from the vortex patch profile, considering briefly the main differences arising between the two profiles in section 3.3.

\subsection{Topographic forcing}

Waves are forced with a topographic perturbation of the form

$$
h_{b}(\lambda, \phi, t)=A(t) \sin ^{2}(2 \phi) \cos \left(2 \lambda+\omega_{0} t\right)
$$

for $\phi>0, h_{b}=0$ for $\phi<0$, comprising a simple zonal wavenumber-two structure in longitude, $\lambda$, with angular frequency $\omega_{0}$ and a compact structure in latitude with maximum amplitude around $45^{\circ} \mathrm{N}$. As in Liu and Scott (2015) and Esler and Scott (2005), the angular frequency $\omega_{0}$ is an external control parameter that may be considered as equivalent to the effect of an arbitrary solid body background rotation, relative to a fixed topography. It thus controls the strength of the polar vortex relative to the topography, with a more negative $\omega_{0}$ corresponding to a stronger vortex. Although the effect of $\omega_{0}$ is felt over the entire atmosphere, its use here is justified in part by the assumption that the vortex stability is determined predominantly by the flow in and around the vortex.

The time dependence of $A(t)$ consists of a gradual increase to a fixed value:

$$
A(t)=A_{0} \cos ^{2}\left(\frac{\pi}{2}(1-t / \tau)\right)
$$




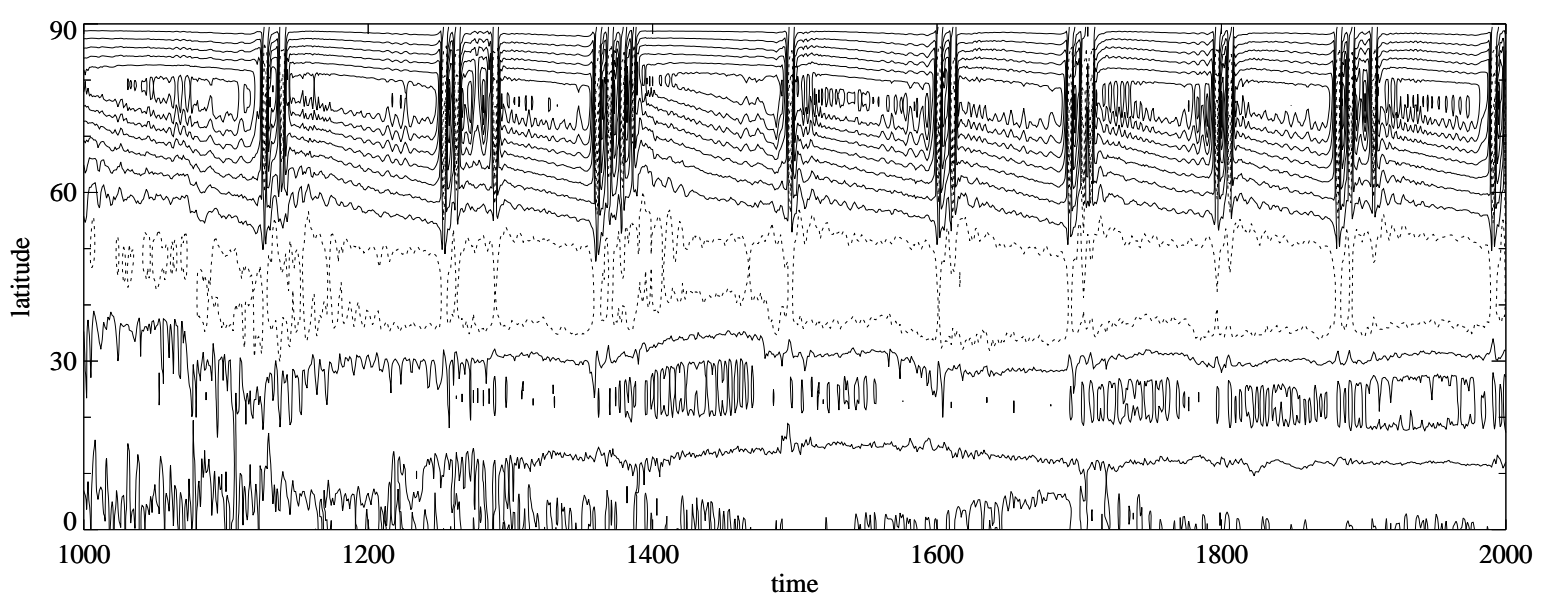

Figure 2. Zonal mean zonal velocity from $t=1000$ days to $t=2000$ days for the case $\omega_{0}=0.0$ and $A_{0}=0.15 H$. Contour interval is $10 \mathrm{~ms}{ }^{-2}$, and negative contours are dotted.

for $t<\tau, A(t)=A_{0}$ for $t \geq \tau$, where the final topographic amplitude $A_{0}$ is a second control parameter. The ramp time, $\tau_{b}$, is taken to be 100 days.

\subsection{Low-latitude momentum relaxation}

As discussed above, form drag arising from the relative westerly flow of the vortex over the topography provides a source of negative total angular momentum that has no systematic sink in the equations (1a)-(1c). To allow a statistically stationary state to be reached while preserving a realistic climatological zonal flow we therefore include a drag on the zonal mean zonal velocity, $\bar{u}$, in the tropics and the southern hemisphere only, restricted away from the northern hemisphere of interest. Specifically, we add to the right-hand side of (1a) a term of the form

$$
-\mathbf{k} \cdot \nabla \times(r(\phi) \bar{u}, 0)
$$

where $r(\phi)$ is the latitude dependent damping rate and takes the form

$$
r(\phi)=r_{0}\left(1-\tanh \frac{\phi-10^{\circ}}{10^{\circ}}\right)
$$

A value of $r_{0}=1 / 20$ days was found sufficient to prevent excessive growth of tropical easterlies across a wide range of the parameters $\omega_{0}$ and $A_{0}$. Note that no drag is applied to the wave component $u^{\prime}=u-\bar{u}$, nor to the meridional velocity $v$.

\subsection{Initial conditions}

In the experiments reported below, it makes no difference whether the initial flow at $t=0$ is in a state of rest or is taken equal to the radiative equilibrium profiles shown in Fig. 1. The flow has little or no memory of the initial conditions beyond about 100 days.

\section{Results}

\subsection{Dynamical characteristics of the vacillations}

The forcing parameters $\omega_{0}$ and $A_{0}$ are varied over ranges $\omega_{0} \in[-0.03,0] 2 \Omega$ and $A_{0} \in[0,0.2] H$. Here we begin by describing the essential dynamical characteristics of the vacillation cycles for the representative values $\omega=0.0$ and $A_{0}=0.15 H$ for the vortex patch equilibrium given by (2). In section 3.2, we summarize the dependence of the vacillations on $\omega_{0}$ and $A_{0}$ and in section 3.3 we briefly describe the main differences arising from the broader equilibrium profile (3).

Figure 2 shows the evolution of the zonal mean zonal velocity for the case $\omega_{0}=0.0$ and $A_{0}=0.15 H$, over the time interval $t=1000$ days to $t=2000$ days. Vacillations are clearly present with a very regularly repeating pattern comprising short episodes of rapid deceleration and reacceleration of the vortex separated by longer intervals in which the vortex strengthens more gradually. As we demonstrate below, the short episodes are dynamic in nature, while the longer intervals are radiative, in the sense that the flow evolution during the short episodes is predominantly adiabatic, while that during the longer episodes is driven by the relaxation term on the right-handside of (1c). The evolution during the radiative intervals is very similar from cycle to cycle. The lengths of the intervals are variable, however, depending in part on the state of the vortex following the dynamic episode.

The dynamic episodes vary in duration and consist of one or more reversals of the zonal mean zonal flow caused by a splitting of the vortex. Figure 3 shows snapshots of the potential vorticity at daily intervals over the time interval between 1598 days and 1617 days. At $t=1600$ the vortex splits in a manner similar to that of the topographic resonant vortex excitation examined in Esler and Scott (2005); Matthewman and Esler (2011); Liu and Scott (2015). Immediately following the vortex split, it undergoes a rapid reformation via the re-merger of the two split lobes, a behaviour noted in the shallow water study of Liu and Scott. During the splitting/merging sequence some of the vortex area is lost to filamentation. In the example shown, the vortex undergoes a second split and re-merger before reducing in size to the extent that resonant excitation is no longer effective. At this point the dynamically controlled episode gives way to the more gradual radiative recovery, during which time the vortex assumes a quasistationary state.

Although the vortex is strongly disturbed during the dynamical episode, and the zonal mean flow changes 

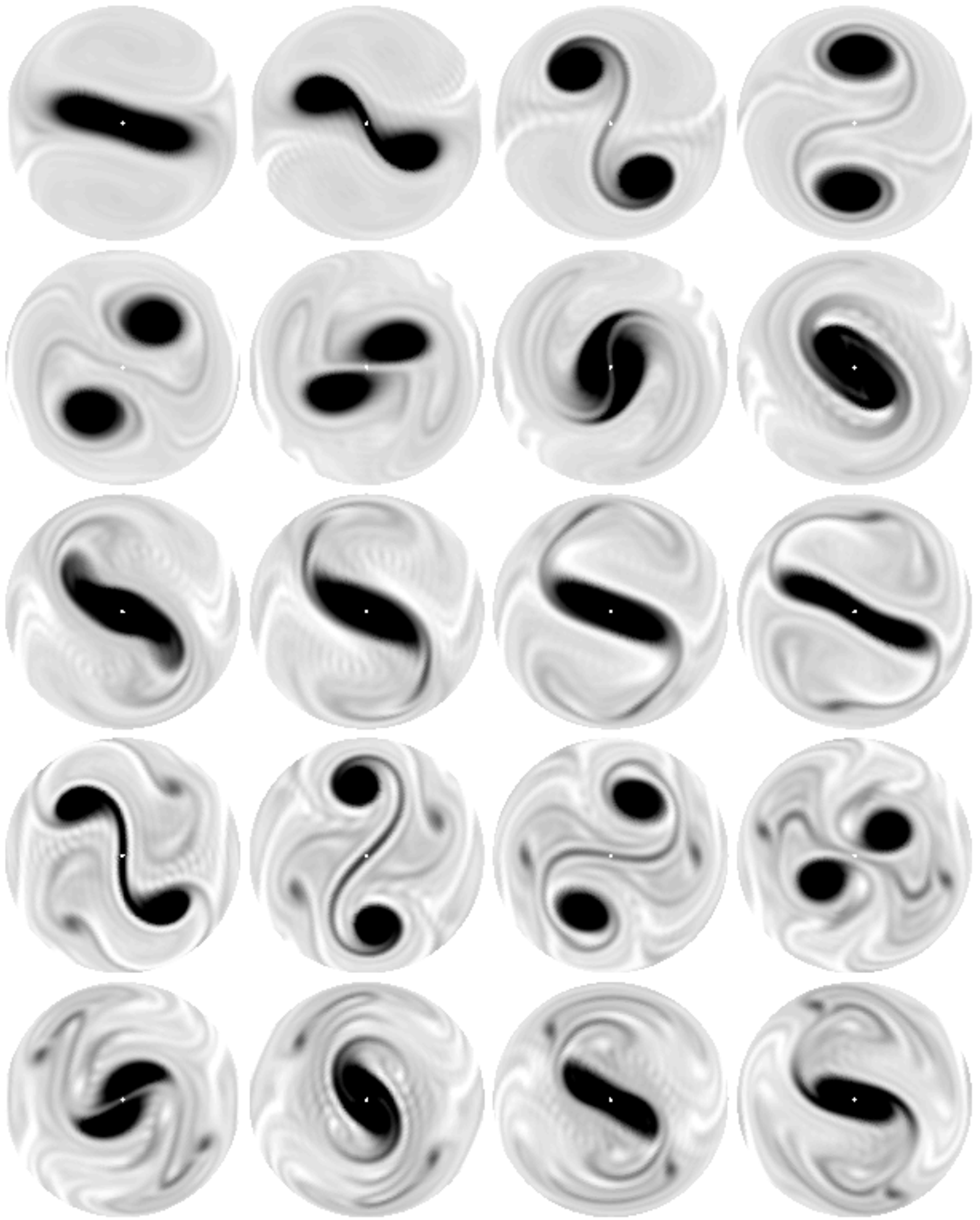

Figure 3. Orthographic projection of the potential vorticity for the case $\omega_{0}=0.0$ and $A_{0}=0.15 H$ at times $t=1598$ days to $t=1617$ days (daily intervals), left-to-right and top-to-bottom.

direction, mixing of the vortex into the surrounding surf zone is far from complete. Over the course of the two vortex split/merger events shown in Figure 3, the vortex loses only about one third of its area to filamentation. In fact, the first splitting event is remarkably reversible, with almost complete re-merger of the two lobes. In an area-based mean, or equivalent latitude framework, the changes to the vortex over the dynamic episode are much less dramatic than in the traditional zonal mean. The equivalent latitude of individual potential vorticity contours are shown in Fig. 4 over a time interval encompasing this and the subsequent dynamic episode. Although the dynamic episodes are visible as a shrinking of the vortex, most of the contours comprising the vortex edge remain in the equivalent latitude band between $70^{\circ}$ and $80^{\circ} \mathrm{N}$ throughout the cycle. Analysis of the terms contributing to the mass tendency of the vortex, as conducted in Rong and Waugh (2004), indicates that the changes in vortex area during the dynamic episode 
are due predominantly to small-scale dissipation due to filamentation, while changes in vortex area during the radiative interval are due predominantly to the diabatic mass flux across the contours. The diabatic source within the vortex itself was found to be an order of magnitude smaller, similar to the findings of both Rong and Waugh (2004) and MirRokni et al. (2011).

The potential vorticity-equivalent latitude evolution can be used to assess the extent to which the vortex evolution is consisent with the resonant excitation of the free mode of the vortex. On the polar $f$-plane, and under quasigeostrophic scaling, Matthewman and Esler (2011) derived the dispersion relation for the free mode as

$$
\frac{\omega_{0}(k, \mathcal{B})}{k}=\frac{Q_{o}}{Q_{i}-Q_{o}} \frac{I_{1}}{\mathcal{B}}+\left(I_{1} K_{1}-I_{k} K_{k}\right)
$$

where $k$ is the zonal wavenumber (here $=2$ ), $\mathcal{B}$ is the vortex radius divided by deformation length, $I_{k}$ and $K_{k}$ are modified Bessel functions or order $k$ evaluated at $\mathcal{B}$, and $Q_{i}$ and $Q_{o}$ are the potential vorticity anomalies (relative to $f$ ) inside and outside the vortex edge, respectively. The two terms on the right-hand side can be considered as representing the strength of the background and the vortex, respectively. Fig. 4 suggests values of $Q_{i}-Q_{o} \approx 1$ and $Q_{o} \approx-0.3$ (taking $f$ to be the Coriolis parameter at the pole) that are approximately constant over the course of the vacillation cycle. The equivalent latitude of the vortex edge varies between about $74^{\circ}$ and $78^{\circ}$, which gives a vortex radius between about $1200 \mathrm{~km}$ and $1600 \mathrm{~km}$, and so, taking $L_{D}=2100 \mathrm{~km}$, values of $\mathcal{B}$ in the range 0.57 to 0.76 . Over this range, $I_{1}(\mathcal{B}) / \mathcal{B} \approx 0.5$ and so the first term on the righthand side of (8) is approximately -0.15 , while the second varies from 0.25 to 0.2 with increasing vortex radius. Thus, as the vortex increases in size during the radiative recovery, the right-hand side decreases towards zero, the condition for resonance. The onset of the split before the right-hand side reaches zero is consistent with a weakly nonlinear self tuning, which further reduces the phase speed of the free mode as the vortex becomes more elliptical (Plumb 1981; Esler and Scott 2005; Matthewman and Esler 2011).

The vortex evolution can be usefully described in terms of its elliptical moments, following Matthewman et al. (2009). In Figure 5, we plot the area (upper), wave amplitude (middle) and excess kurtosis (lower) based on the PV contour $q=2 \Omega$, which is located in the vortex edge throughout the evolution (as indicated by the bold contour in Fig. 4). The wave amplitude is closely related to the quasigeostrophic wave activity and provides a meaningful measure of the departure of the vortex from an axisymmetric state. The sharp peaks in wave activity coinciding with the vortex splitting provide further evidence that the splits are due to resonant growth, rather than, for example, a gradual migration of the vortex over the topography. The excess kurtosis is a measure of the departure of the vortex shape from an ellipse: positive values correspond to an eye shape with the vortex narrowing at its extremities, large values typically associated with filamentation of vortex material into the surf zone; negative values correspond to a dumbbell shape with the vortex pinching in the middle, values below about -0.5 typically resulting in complete splitting of the vortex. The reader is referred to Liu and Scott (2015) (section 2.5, therein) for the definitions of both quantities in terms of second and

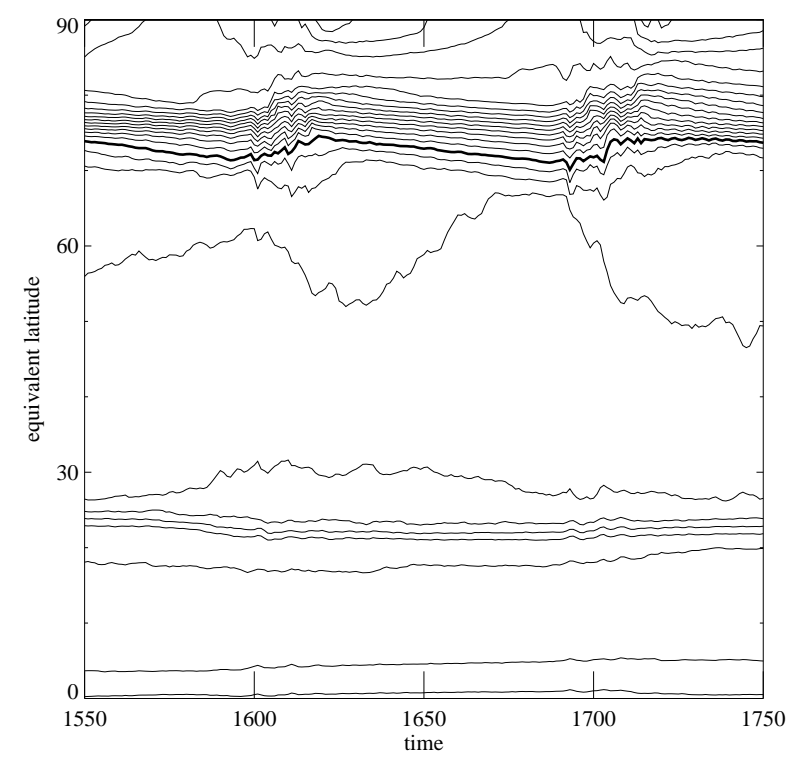

Figure 4. Potential vorticity as a function of equivalent latitude and time. Contour interval is $0.1 \times 2 \Omega$ and the level $q=2 \Omega$ is shown bold.

fourth order moments and their method of computation on the sphere.

The vortex splitting events during the dynamic episodes are captured most clearly by the excess kurtosis. Most episodes consist of two or three repeated splits; the one beginning just before $t=1400$ days involves as many as four, while the one at $t=1500$ days involves only one. Many, but not all, vortex splits are followed by a large growth of positive kurtosis, indicating further filamentation of the vortex from its extremities: an example occurs around $t=1400$ days, when the dynamic episode finishes with a prolonged interval of filamentation and significant area loss (top panel). In contrast, the dynamic episode between $t=1250$ and 1300 days involves three clear vortex splits but very little filamentation and area loss of the vortex. Other time intervals and different forcing parameters reveal similar variation in number and details of splitting events. The dynamics during this time appears to be chaotic and influenced by small details in the configuration of the vortex and its surroundings.

The intervals of radiative recovery, in contrast, are very regular. They involve both a gradual growth in the vortex area (see also Fig. 4) and a simultaneous growth in the vortex wave amplitude. Note that the excess kurtosis remains very nearly zero throughout this period reflecting the fact that the vortex remains elliptical to a very good approximation. The orientation of the ellipse remains approximately constant throughout this interval (oriented approximately left-to-right as in the first and last panels of Fig. 2).

\subsection{Dependence on forcing parameters}

The variability shows a complicated dependence on forcing amplitude $A_{0}$. Over the parameter ranges considered, the dependence on the forcing frequency $\omega_{0}$ is relatively weak and we focus therefore on the case $\omega_{0}=0.0$ discussed in the previous section.

As can be expected, when the forcing amplitude is very weak, the vortex adjusts into a stable and steady equilibrium 


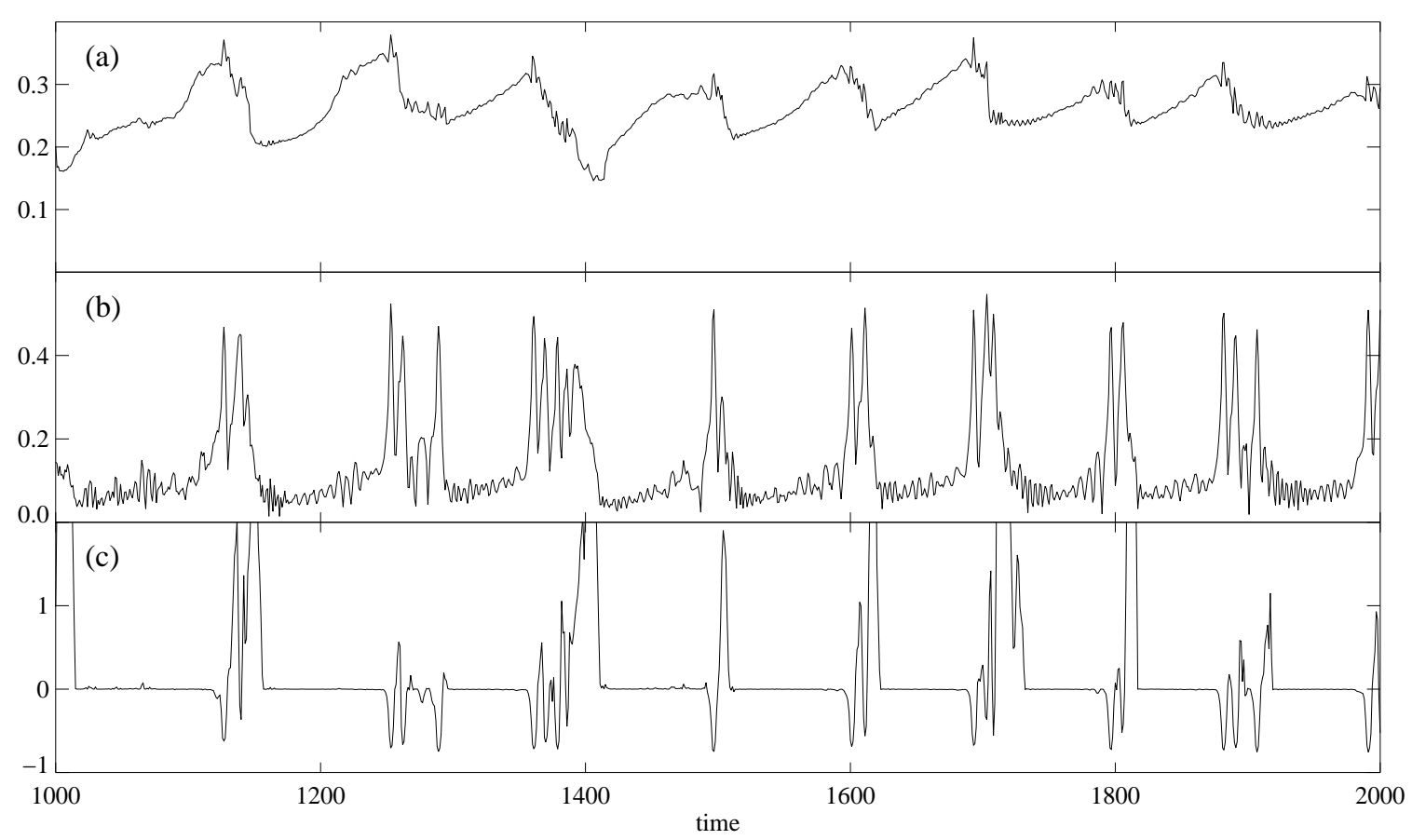

Figure 5. Elliptic diagnostics based on the PV level $q=2 \Omega$ : (a) vortex area, (b) wave amplitude (c) excess kurtosis.

state of approximately elliptic shape, and oriented along the extending axes of the strain field induced by the topography. The states are similar to the steady equilibrium state shown in Fig.4 of Liu and Scott (2015). This state is replaced by vacillating states similar to that described above when the forcing amplitude exceeds a value of about $0.05 \mathrm{H}$. The subsequent dependence on $A_{0}$ is summarized in Figure 6, which shows the excess kurtosis for the extended period $t=$ 500 to $t=2500$ days for forcing amplitudes $A_{0}=0.05 \mathrm{H}$ to $A_{0}=0.25 H$. The vacillating states persist as the forcing amplitude is increased to the value $0.15 H$, corresponding to the case just examined. Over this range there is very little systematic dependence of the details of the vacillation cycles on the forcing amplitude beyond a slight increase in period with increasing $A_{0}$. On the other hand, there are quite large variations in the details of individual vacillation cycles.

Beyond $A_{0}=0.15 H$ the forcing is sufficiently strong that the vortex becomes strongly disturbed for more prolonged time intervals, indicated in the plots of excess kurtosis by dotted lines. These weak vortex intervals are defined as intervals in which the root mean square zonal mean zonal velocity, averaged over the polar cap and smoothed in time with a median smoothing using a 60 day window, drops below $20 \mathrm{~ms}^{-1}$. These intervals first appear at $A_{0}=0.175 H$ and generally become longer as forcing amplitude increases further (lower four panels of Fig. 6). Remarkably, however, even after prolonged periods in which the vortex is essentially absent, the flow may spontaneously revert to a coherent vacillating vortex, which may subsequently persist for many cycles. An example of such a regime change from weak to strong vacillating vortex occurs for $A_{0}=0.2 \mathrm{H}$ at around $t=1700$ days (Fig. 6, sixth panel down). The evolution of the zonal mean zonal velocity before and following the transition is shown in Fig. 7, revealing the spontaneous re-development of the vortex and onset of the vacillations from a prolonged state of near zero flow. Such regime changes are found over a range of forcing amplitudes between $A_{0}=0.175 H$ to $A_{0}=$ $0.25 H$, but at higher values of the forcing the intervals over which the vortex is weak increase in length as the forcing prevents the re-establishment of the vacillation regime.

Excluding the regime transitions, over the entire forcing range from $A_{0}=0.05 H$ to $0.25 H$, there is a general increase in the vacillation period with increasing $A_{0}$. This may be contrasted to the case of vacillations obtained previously in models with vertical structure, which tended to show a reduction in period with increasing forcing amplitude, following a Hopf-type bifurcation (Christiansen 2000). Here, the increase of period with $A_{0}$ appears to be due to an increase of area loss during the dynamic episode, and a consequent longer recovery period. Note that since the vortex split occurs as a resonance of the vortex with the forcing, the onset of the splitting is controlled to a greater extent by the state of the vortex than the forcing amplitude.

Frequency spectra of the zonal mean wind near the vortex edge (not shown) have a bimodal distribution with a maximum around periods of 10 days due to the splitting and merger processes and a separate maximum around periods of 100 days arising from the period of the entire vacillation cycle. At larger forcing amplitudes, the low-frequency peak is reddened significantly by the prolonged intervals in which the vortex is absent. Beyond the double peak structure, the spectra do not reveal any features not already apparent in the zonal mean and elliptic diagnostics considered above.

\subsection{Dependence on equilibrium height field}

We close with a brief description of vacillation cycles obtained with the alternative equilibrium height profile given by (3) and shown in Fig. 1b. This profile is very similar to the one used by Rong and Waugh (2004) and MirRokni et al. (2011), but is smoother at low latitudes to reduce a discontinuity and non-monotonicity in the 


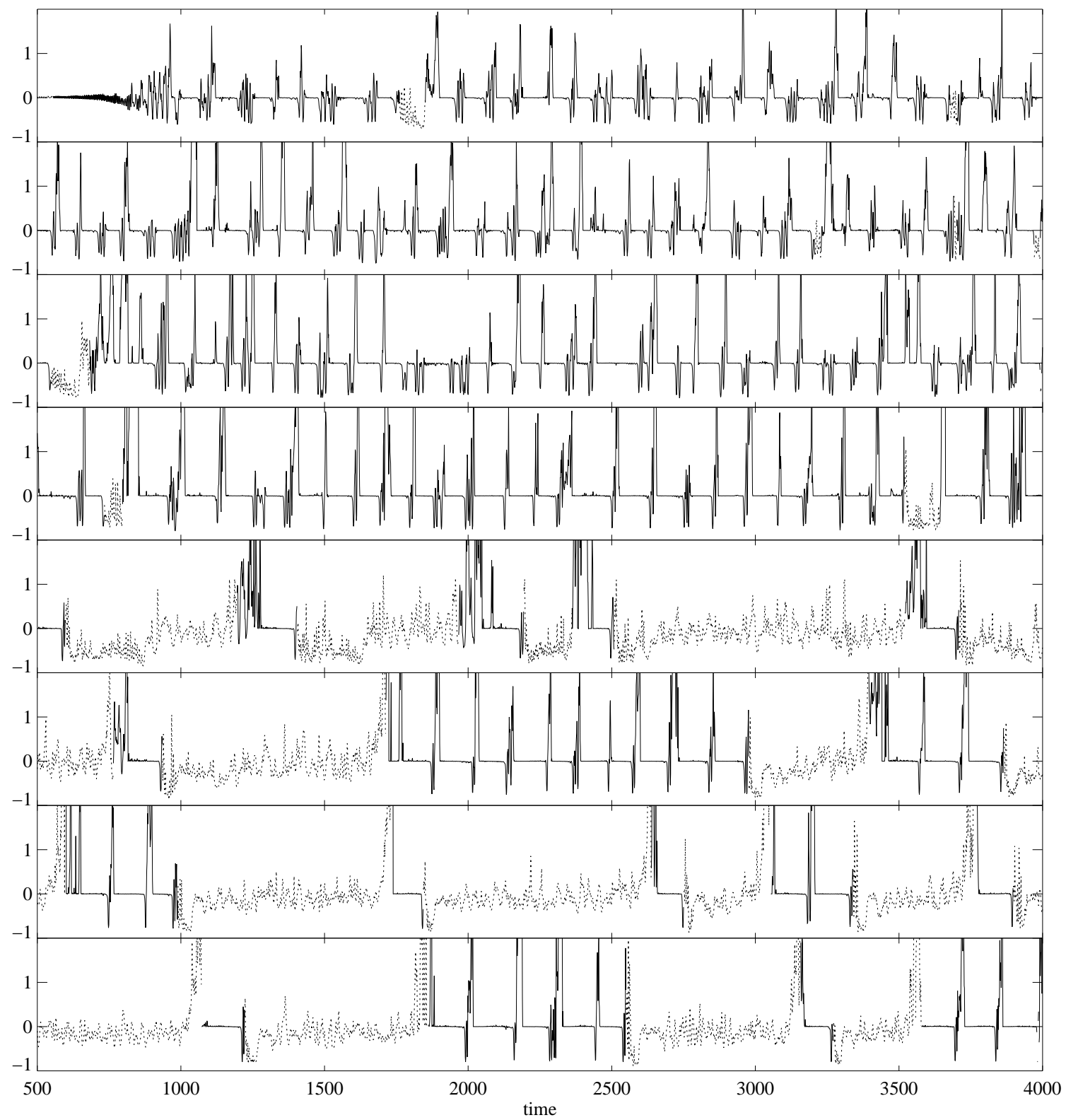

Figure 6. Excess kurtosis based on the PV level $q=2 \Omega$ for cases $A_{0}=0.075 H, A_{0}=0.1 H, \ldots, A_{0}=0.225 H, A_{0}=0.25 H$ (top to bottom) Dotted lines indicate weak vortex regimes, defined as times during which the median rms zonal velocity north of $70^{\circ}$ falls below 20 ms ${ }^{-1}$, where the median is calculated over an interval of 60 days.

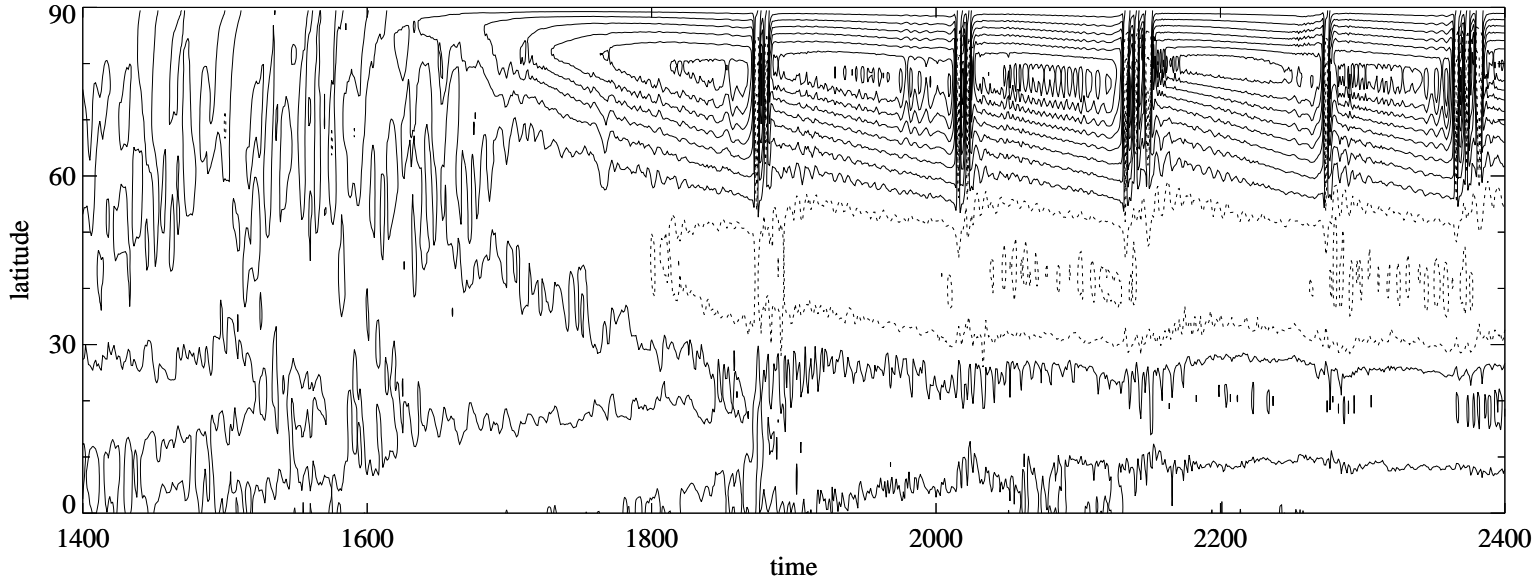

Figure 7. Zonal mean zonal velocity from $t=1400$ days to $t=2400$ days for the case $\omega_{0}=0.0$ and $A_{0}=0.2 H$. Contour interval is $10 \mathrm{~ms}-2$, and negative contours are dotted. 
potential vorticity at $10^{\circ} \mathrm{N}$. The main difference from the patch equilibrium considered in the previous discussion is that the potential vorticity has a much broader profile with no well-defined vortex edge. Despite this difference, the evolution of the flow is remarkably similar to that obtained with the patch equilibrium, partly due to the well-known tendency of such smooth initial distributions to develop well-defined potential vorticity gradients at the vortex edge and in the substropics (Juckes and McIntyre 1987; Polvani et al. 1995).

The zonal mean zonal velocity for the case $\omega_{0}=-0.02$ and $A_{0}=0.1 H$, over the time interval $t=1000$ days to $t=2000$ days, is shown in Fig. 8a. The flow exhibits vacillations of the vortex similar to those shown in Fig. 2, again consisting of short, dynamically controlled episodes of zonal flow reversal, separated by longer radiatively controlled intervals of vortex recovery. The main differences between this case and the one shown in Fig. 2 lie in the low-latitude flow, which exhibits greater lowfrequency variability with the broader equilibrium profile. The development and maintenance of the sharp polar jet around $70^{\circ}$ is a striking example of the mechanism of jet self-sharpening (McIntyre 1982; Juckes and McIntyre 1987; Dritschel and Scott 2011). Plots of potential vorticity against equivalent latitude indicate that the formation of a sharp vortex edge is a generic feature of these simulations.

Figure. 9 shows snapshots of the potential vorticity during the dynamical episode around time $t=1700$ days. The main feature of the episode is again the sudden vortex split and re-merger event occurring over the space of a few days ( $t=1709-1714$ days). The main difference is that here the split is preceded by an interval of vortex erosion, in which the potential vortex gradients near the vortex are intensified into a more patch-like distribution. This interval may be considered as a preconditioning of the vortex, as commonly observed in the winter stratosphere, that brings the vortex closer to a state of resonance with the forcing. Here, it is a robust component of the vacillation cycle and occurs before the splitting events of other dynamical episodes. Thus, the potential vorticity profile just before splitting is not controlled directly by the radiative equilibrium state, but as a possible rearrangement of that state into a staircase-like profile (Dunkerton and Scott 2008).

The dependence of the vacillations on the forcing parameters $A_{0}$ and $\omega_{0}$ shows no significant differences from before, although a more negative value of $\omega_{0}$ was required here to obtain regular cycles. The more negative values of $\omega_{0}$ are again consistent with the suggestion that the vortex splitting is associated with the resonant excitation of the free vortex mode. Specifically, a slightly larger vortex area here, compared with the case considered in section 3.1, results in the second term in (8) being reduced slightly by an amount, very approximately, of the order 0.01-0.02. At larger values of $A_{0}$ a mixture of weak vortex and strong, vacillating vortex regimes were again obtained with sudden regime transitions between the two; an example is shown in Fig. $8 \mathrm{~b}$ for the case $A_{0}=0.175 H$.

\section{Summary and conclusions}

It appears that the inability of previous studies of the forced-relaxed shallow water equations to obtain persistent vacillations over long time intervals and at high horizontal resolution is due mainly to the unconstrained growth of easterly zonal winds in the tropics. The source of negative global angular momentum arising from the topographic forcing and associated form drag has no systematic sink when relaxation is applied to the height field only. Relaxation on the height field constrains local angular momentum in the extra tropics through thermal wind balance, meaning that the increase in negative global angular momentum manifests as unrealistically strong tropical easterlies. In this paper, a local angular momentum sink has been applied in the tropics, which effectively allows a statistically stationary state to be reached in which the input of negative angular momentum due to the topographic forcing is balanced by the tropical sink. Under these conditions, we have demonstrated that persistent and regular vacillations arise robustly in the shallow water model, under a wide range of forcing and relaxation conditions. In particular, dynamically similar vacillations are obtained for two very different equilibrium height fields, one corresponding to a potential vorticity patch with sharp vortex edge, the other to a hemispherically broad and smooth potential vorticity distribution.

The vacillation cycles obtained show a number of notable differences from vacillations previously obtained in primitive equation and earlier height dependent models. First, while the splitting of the vortex at the onset of the dynamical episode is similar to the behaviour found in Scott and Polvani (2006), an important aspect of the evolution found here is the rapid remerger of the two resulting vortex lobes. Because of this rapid remerger, during which time the vortex may lose only a small fraction of its total area, the vortex may continue to split and remerge several times before the transition to the radiatively controlled recovery stage. Second, the radiative recovery itself differs in the more obvious regard of vertical wave propagation. Primitive equation vacillation cycles exhibit a clear vertical structure in the evolution of the zonal mean zonal wind, with upper-level vortex recovery while weak low-level winds inhibit strong wave growth. Here the vortex recovers simply because it is not close to resonance with the topographic forcing. Finally, an interesting property of the system considered here is the presence of distinct dynamical regimes, separated by rapid transitions that appear to occur at much lower frequencies than those of the vacillation cycles themselves. At higher forcing amplitudes, the flow may enter a state in which the polar vortex is almost completely suppressed by the wave field induced by the topography. From such a state it may recover spontaneously to one of a strong, vacillating vortex similar to those found at lower amplitudes. Such regime transitions were not observed in the primitive equation experiments of Scott and Polvani (2006).

The rapid vortex merger seen above is not a typical feature of observed vortex splitting stratospheric sudden warming events. The composite vortex splitting major warmings shown in Figure 4 of Matthewman et al. (2009) suggest that the two lobes remain well-separated at least 5 days following the time of the split. Nonetheless, it is interesting to note that re-merger is indeed observed in certain sudden warming events, in particular if we consider also events that fail to meet the usual criterion of major warming, yet still exhibit vortex splitting. As an example, Fig. 10 shows daily snapshots of potential vorticity on the $600 \mathrm{~K}$ isentropic surface for the period January 1-January 8, 2015, from the ERA-interim data set. The zonal mean zonal velocity over this period (not shown) does not completely 


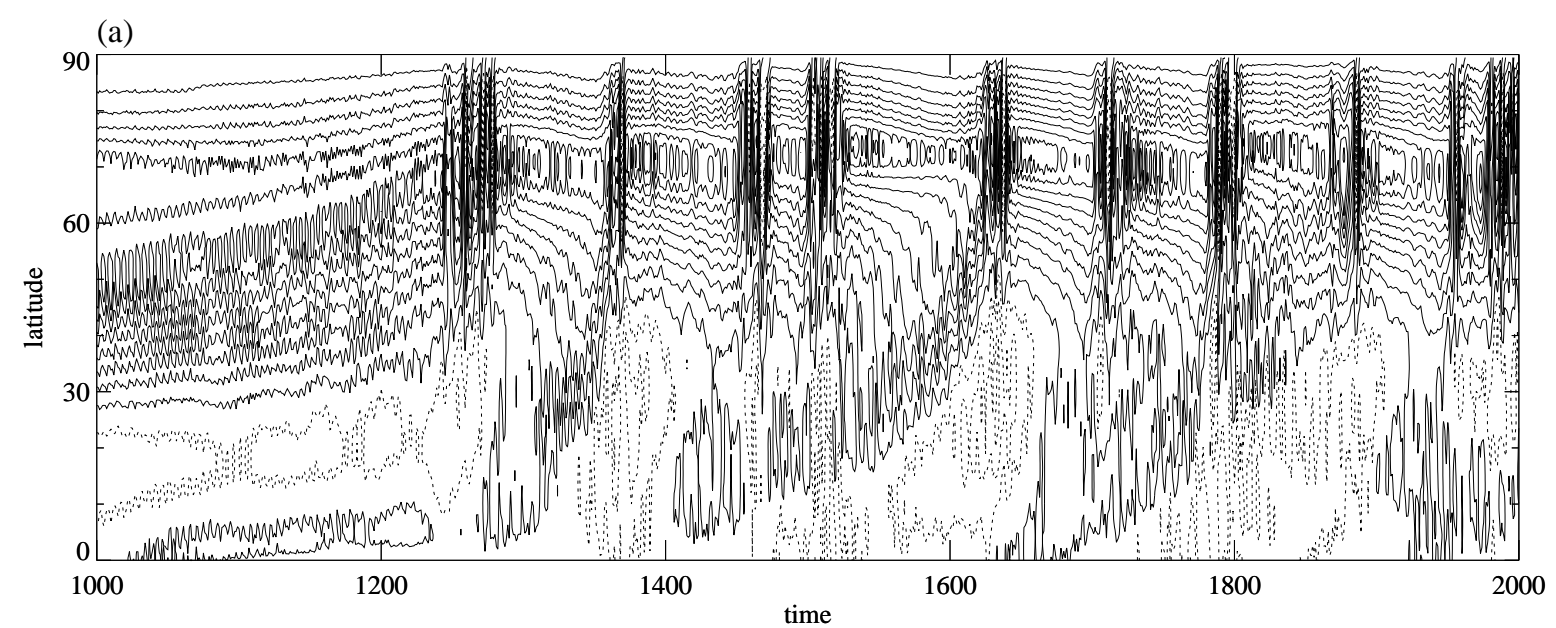

(b)

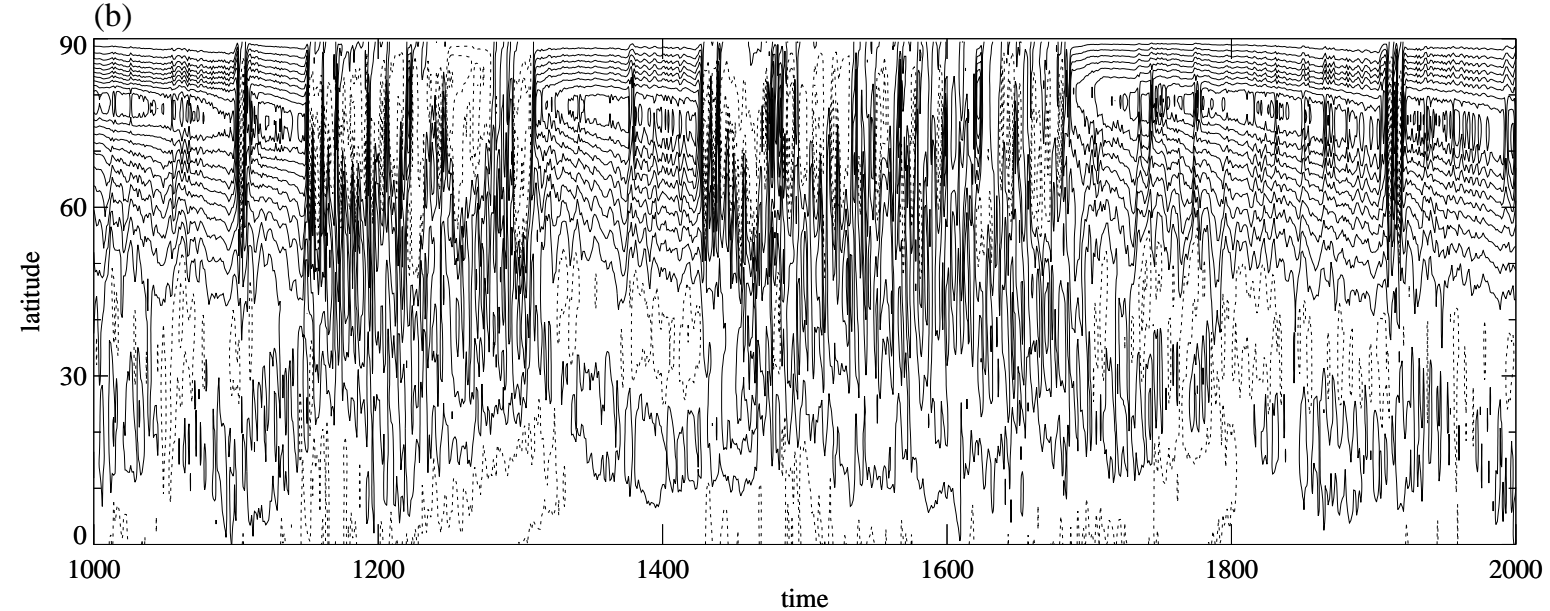

Figure 8. Zonal mean zonal velocity from $t=1000$ days to $t=2000$ days for the cases $\omega_{0}=-0.02$ and $A_{0}=0.1 H$ (top) and $A_{0}=0.175 H$ (bottom), obtained with the equilibrium height field given by (3). Contour intervals as in Fig. 7.

reverse, meaning that this event was not classified as a major warming, despite the clear split of the vortex around January 2-3. Although the evolution here is complicated by the presence of other planetary-scale waves, in particular a wavenumber one mode that displaces the vortex from over the pole, the re-merger of the two lobes over the course of the following four days is not dissimilar to the evolution of the single layer model discussed above. Other vortex splitting sudden warming events that exhibit remerger include ones in January 1985 and January 2003 (not shown). In the 2003 event, the vortex evolves in a way very similar to that shown in Fig. 10, where the splitting is such that the resulting lobes are only weakly separated and subsequent re-merger is favoured. In the 1985 splitting, the resulting lobes are more widely separated, yet the configuration of the background flow results in almost complete remerger a few days later.

Clearly whether two lobes resulting from a vortex splitting event subsequently remerge or are instead subject to irrevesible mixing into the surf zone has a major influence on stratospheric transport that may be difficult to quantify using traditional Eulerian zonal mean quantities. Alternative criteria for the classification of sudden warmings have been put forward based on the geometry of the vortex by, for example, Waugh and Randel (1999) and Seviour et al. (2013), and such criterion have been useful in analysing circulation anomalies in the troposphere following sudden warming events (e.g. Mitchell et al. 2013). Extended radiatively controlled regimes following sudden warming events have been found to occur in nature and have been suggested as possible sources of increased skill in tropospheric seasonal forecasting (Hitchcock et al. 2013). The present results suggest that whether the vortex enters such a radiatively dominated regime is not completely determined by the kurtosis exceeding the threshold necessary for splitting, but is also closely related to the amount of area loss incurred by the vortex subsequent to the sudden warming itself.

\section{Acknowledgements}

The author wishes to express his thanks to two anonymous reviewers for helpful and insightful comments on the original version of the manuscript.

\section{References}

Chao WC. 1985. Sudden stratospheric warmings as catastrophes. $J$. Atmos. Sci. 42: 1631-1646.

Christiansen B. 1999. Stratospheric vacillations in a general circulation model. J. Atmos. Sci. 56: 1858-1872.

Christiansen B. 2000. Chaos, quasiperiodicity, and interannual variability: studies of a stratospheric vacillation model. J. Atmos. Sci. 57: 3161-3173.

Dritschel DG, Scott RK. 2011. Jet sharpening by turbulent mixing. Phil. Tran. Roy. Soc. A 369: 754-770. 

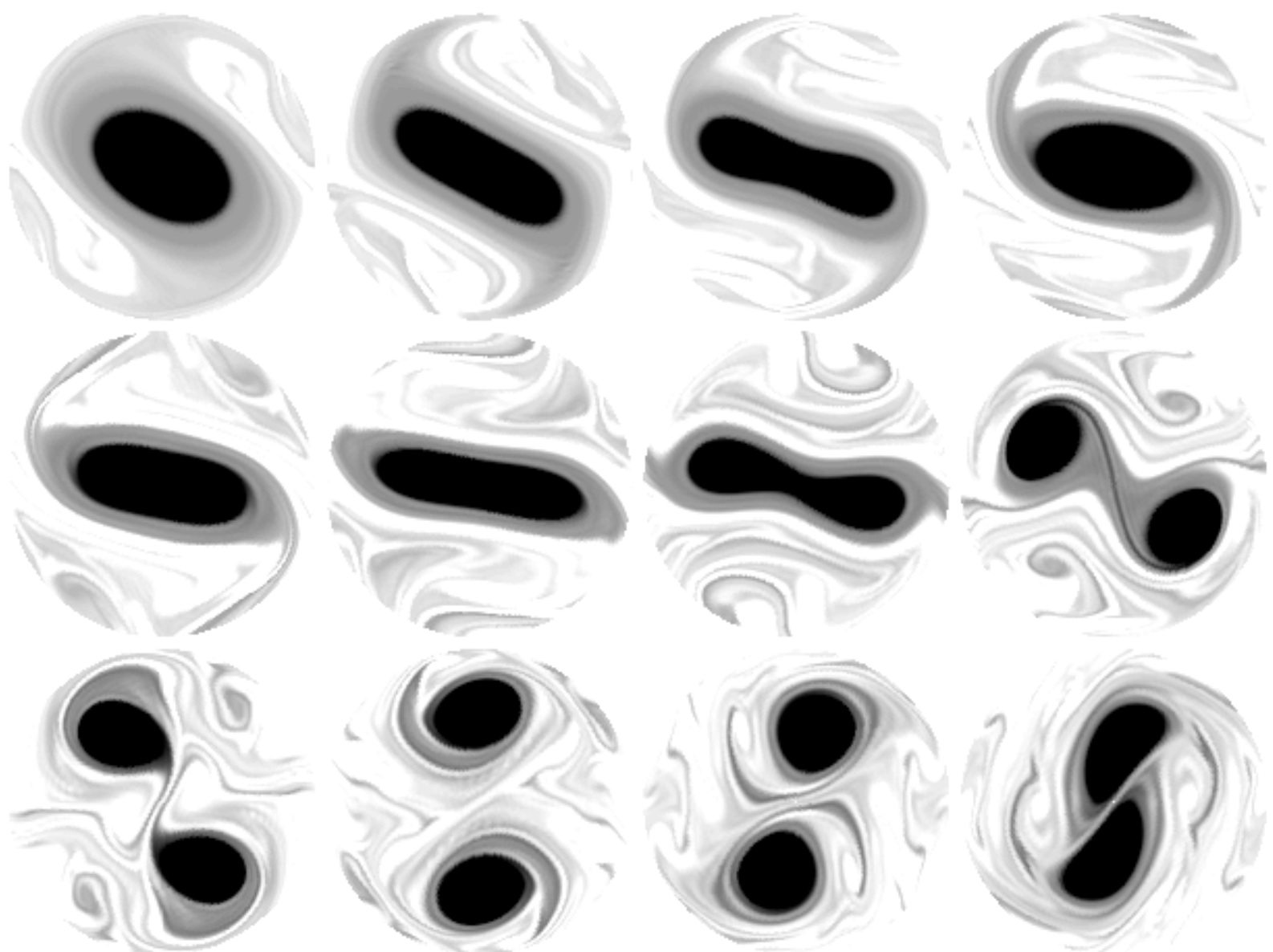

Figure 9. Orthographic projection of the potential vorticity for the case $\omega_{0}=-0.02$ and $A_{0}=0.1 H$, obtained with the equilibrium height field given by (3); times $t=1700$ days to $t=1706$ days in two-day intervals (left to right, top), and $t=1707$ days to $t=1714$ days in daily intervals (left to right, middle to bottom).
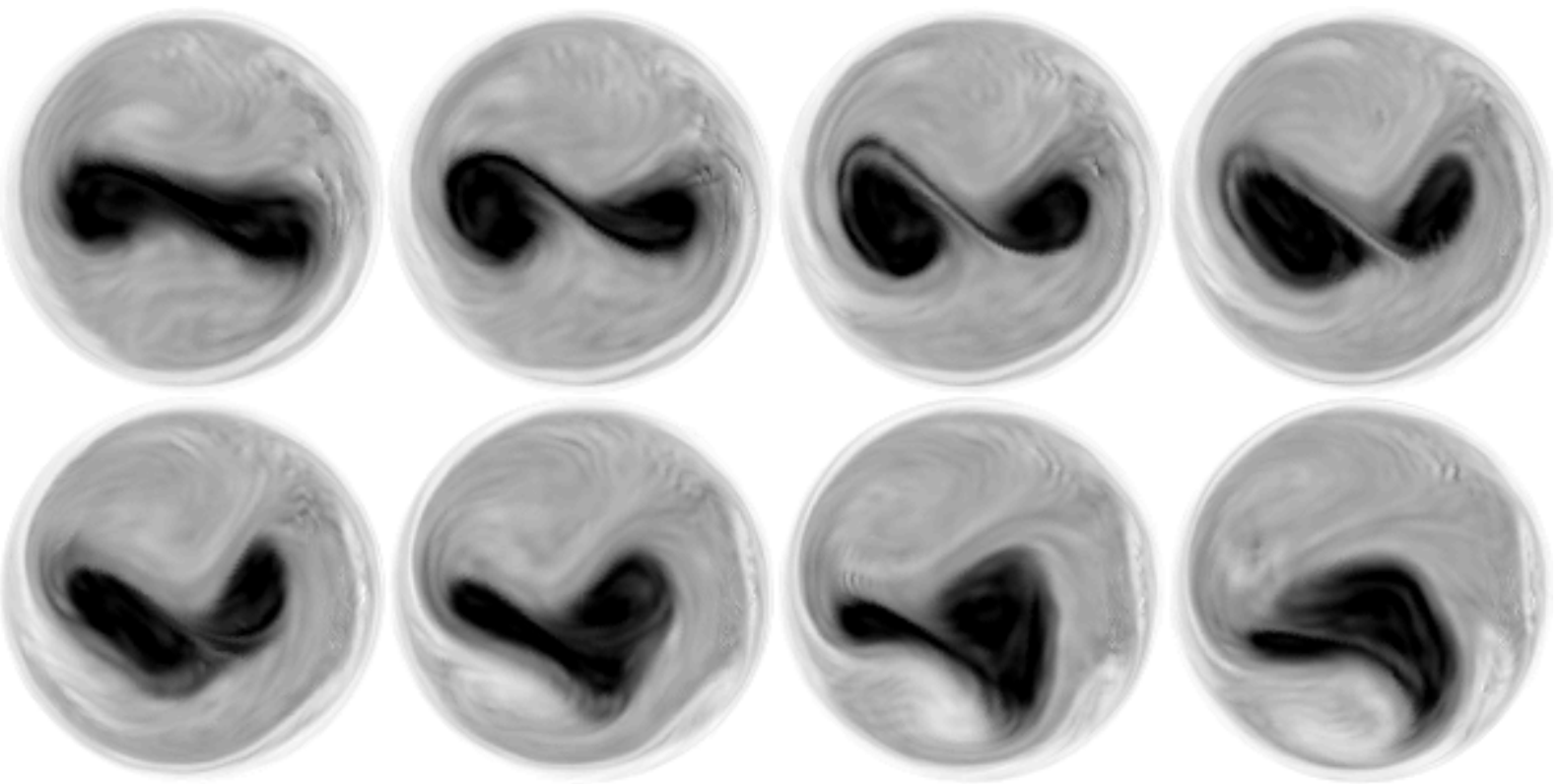

Figure 10. Potential vorticity on the $600 \mathrm{~K}$ potential temperature level, at daily intervals at 0000 UTC, January 1-January 8 , 2015 (left to right, top to bottom), from the ERA-interim data set; data courtesy of ECMWF.

Dunkerton TJ, Scott RK. 2008. A barotropic model of the angular momentum conserving potential vorticity staircase in spherical geometry. J. Atmos. Sci. 65: 1105-1136.

Esler JG, Scott RK. 2005. On the excitation of transient Rossby waves on the polar stratospheric vortex and the barotropic sudden warming. J. Atmos. Sci. 62: 3661-3682.
Hitchcock P, Shepherd TG, Manney GL. 2013. Statistical characterization of arctic polar-night jet oscillation events. J. Clim. 26: 20962116.

Holton JR, Dunkerton T. 1978. On the role of wave transience and dissipation in stratospheric mean flow vacillations. J. Atmos. Sci. 35: 
74-744.

Holton JR, Mass C. 1976. Stratospheric vacillation cycles. J. Atmos. Sci. 33: 2218-2225.

Juckes MN, McIntyre ME. 1987. A high resolution, one-layer model of breaking planetary waves in the stratosphere. Nature 328: 590-596.

Liu YS, Scott RK. 2015. The onset of the barotropic sudden warming in a global model. Q. J. Roy. Meteor. Soc. : doi: 10.1002/qj.2580.

Matthewman NJ, Esler JG. 2011. Stratospheric Sudden Warmings as Self-Tuning Resonances. Part I: Vortex Splitting Events. J. Atmos. Sci 68: 2481-2504.

Matthewman NJ, Esler JG, Charlton-Perez AJ, Polvani LM. 2009. A new look at stratospheric sudden warmings. Part III. Polar vortex evolution and vertical structure. J. Clim. 22: 1566-1585.

McIntyre ME. 1982. How well do we understand the dynamics of stratospheric warmings? J. Meteorol. Soc. Japan 60: 37-65. Special issue in commemoration of the centennial of the Meteorological Society of Japan, ed. K. Ninomiya.

MirRokni SM, Mohebalhojeh AR, Dritschel DG. 2011. Revisiting vacillations in shallow-water models of the stratosphere using potential-vorticity-based numerical algorithms. J. Atmos. Sci. 68 : 1007-1022.

Mitchell DM, Gray LJ, Anstey J, Baldwin MP, Charlton-Perez AJ. 2013. The influence of stratospheric vortex displacements and splits on surface climate. J. Clim. 26: 2668-2682.

Plumb RA. 1981. Instability of the distorted polar night vortex: a theory of stratospheric warmings. J. Atmos. Sci. 38: 2514-2531.

Polvani LM, Waugh DW, Plumb RA. 1995. On the subtropical edge of the stratospheric surf zone. J. Atmos. Sci. 52: 1288-1309.
Rivier L, Loft R, Polvani LM. 2002. An efficient spectral dynamical core for distributed memory computers. Mon. Weather Rev. 130: 1384 1390.

Rong PP, Waugh DW. 2004. Vacillations in a shallow-water model of the stratosphere. J. Atmos. Sci. 61: 1174-1185.

Scott RK, Haynes PH. 2000. Internal vacillations in stratosphere-only models. J. Atmos. Sci. 57: 2333-2350.

Scott RK, Liu YS. 2015. On the formation and maintenance of the stratospheric surf zone as inferred from the zonally averaged potential vorticity distribution. Q. J. Roy. Meteor. Soc. 141: 327-332.

Scott RK, Polvani LM. 2006. Internal variability of the winter stratosphere. Part I: Time independent forcing. J. Atmos. Sci 63: 2758-2776.

Scott RK, Rivier L, Loft R, Polvani LM. 2004. BOB: Model implementation and users guide. NCAR Technical Note : 30ppNCAR/TN456+IA.

Seviour WJM, Mitchell DM, Gray LJ. 2013. A practical method to identify displaced and split stratospheric polar vortex events. Geophys. Res. Lett. 40: 5268-5273.

Thuburn J, Langneau V. 1999. Eulerian mean, contour integral, and finite-amplitude wave activity diagnostics applied to a single-layer model of the winter stratosphere. J. Atmos. Sci 56: 689-710.

Waugh DW, Randel WJ. 1999. Climatology of Arctic and Antarctic polar vortices using elliptical diagnostics. J. Atmos. Sci. 56: 1594 1613.

Yoden S. 1987. Dynamical aspects of stratospheric vacillations in a highly truncated model. J. Atmos. Sci. 44: 3683-3695.

Yoden S. 1990. An illustrative model of seasonal and interannual variations of the stratospheric circulation. J. Atmos. Sci. 47: 18451853 